an inflammatory disease (overlap, infection, neoplasia), and showed a tendency to an association with mortality. This association became statistically significant when considering DD plasma levels as a quantitative variable $(p<0.001)$ and remained significant after adjustments (age, coexistence of an inflammatory disease) (p 0,003).

Conclusions: DD plasma levels are associated with macrovascular involvement and can be helpful in predicting medium-term mortality in our SSc patients. Levels of plasma DD can be modified by systemic inflammation.

Disclosure of Interest: None declared

DOI: 10.1136/annrheumdis-2018-eular.7504

\section{SAT0496 INCIDENCE, CARDIOVASCULAR EVENTS AND MORTALITY OF ADULT INFLAMMATORY MYOPATHIES IN SOUTH KOREA: A NATIONWIDE POPULATION- BASED STUDY}

K.H. Jung ${ }^{1}$, H.J. Kim², W. Park ${ }^{1}$, S.R. Kwon ${ }^{1}$, H.S. Ahn ${ }^{2} .{ }^{1}$ Rheumatology, INHA University, Incheon; ${ }^{2}$ Preventive medicine, Korea University, Seoul, Korea, Republic Of

Background: The inflammatory myopathies (IMs), including dermatomyositis (DM) and polymyositis (PM), are rare autoimmune diseases characterised by progressive proximal muscle weakness, elevated muscle enzyme and various organ involvement. Studies on epidemiology and mortality of IMs at the national level are rare.

Objectives: We conducted a nationwide population study of incidence, cardiovascular events (especially acute myocardial infarction, ischaemic stroke, haemorrhagic stroke) and survival of IMs in South Korea over the course of 11 years. Methods: We used data from the Rare Intractable Disease (RID) registry and Health Insurance Review and Assessment (HIRA) service, which include information on all IMs patients diagnosed based on uniform criteria between 2005 and 2015. Survival data from Statistics Korea linked to HIRA-RID database were used in our survival analysis.

Results: In this study, total incident cases between 2005 and 2015 were 3014 (1,860 DM patients, 1,154 PM patients) and the mean annual incidence rate was $7.16 / 10^{6} /$ year (DM was $4.42 / 10^{6} /$ year, PM was $2.74 / 10^{6} /$ year). The female to male ratio of DM was 2.2:1 and that of PM was 1.9:1. Cardiovascular events occurred in 155 patients during the study period. Of the patients with DM, 25 were diagnosed with AMI (SIR 1.3, 95\% Cl 0.9-1.9), 39 with ischaemic stroke (SIR 1.4, $95 \% \mathrm{Cl} 1.0-1.9$ ), 18 with haemorrhagic stroke (SIR $1.3,95 \% \mathrm{Cl} 0.8-2.0)$. The results of PM were similar with those of DM. Of 155 patients, $63(40.6 \%)$ died and had a high mortality rate. 640 of 3014 patients with IMs died during the study period; 290 and 350 were male and female, respectively. The survival curve for IMs is shown in Fig.1. The DM patients showed a 5 year survival rate (YSR) of $76.8 \%$, and a $10-Y S R$ of $68.9 \%$, and the PM patients showed a $5-Y S R$ of $79.3 \%$ and a $10-Y S R$ of $68.3 \%$.

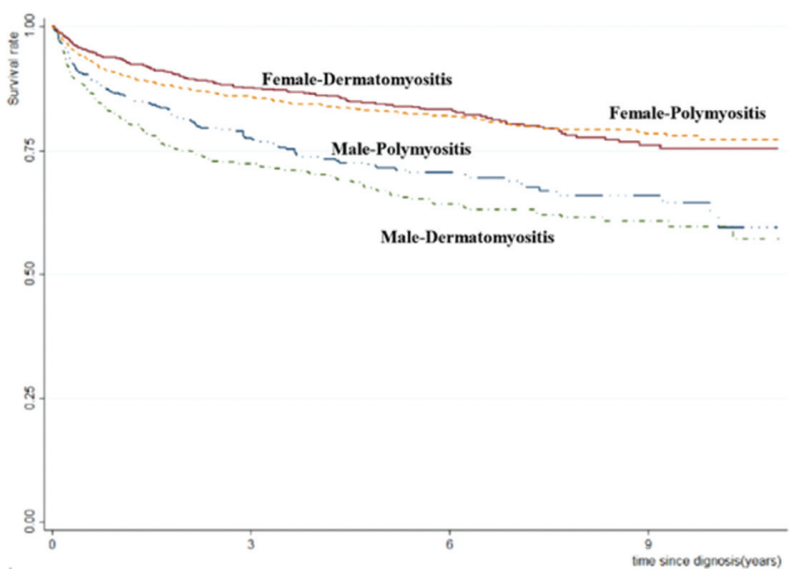

Abstract SAT0496 - Figure 1. Survival curves of dermatomyositis and polymyositis

Conclusions: This is the first study of incidence and mortality of IMs over 3000 cases by the national registry in South Korea. In this nationwide population-based study of IMs, we found still high mortality and low survival and increased cardiovascular events related mortality compared than general population.

Disclosure of Interest: None declared

DOI: 10.1136/annrheumdis-2018-eular.5564

\section{SAT0497 \\ FOLLOW-UP OF DISEASE ACTIVITY IN HUNGARIAN PATIENTS WITH IDIOPATHIC INFLAMMATORY MYOPATHY}

L. Bodoki ${ }^{1}$, M. Nagy-Vincze ${ }^{2}$, Z. Griger ${ }^{2}$, Z. Szekanecz ${ }^{1}$, K. Dankó ${ }^{2}$. ${ }^{1}$ University Of Debrecen, Faculty Of Medicine, Department Of Internal Medicine, Department Of Rheumatology, ${ }^{2}$ University Of Debrecen, Faculty Of Medicine, Department Of Internal Medicine, Department Of Clinical Immunology, Debrecen, Hungary

Background: Assessing disease activity in patients with myositis is an important goal. A method called Disease Activity Core Set Measures was established by International Myositis Assessment and Clinical Studies Group (IMACS) for evaluating activity of myositis. This assesses the manifestations of myositis which are thought to be reversible that result directly from the inflammatory process.

Objectives: In this prospective study authors aimed to monitoring disease activity in Hungarian myositis patients treated at the Autoimmune Outpatient Clinic Department of Clinical Immunology, University of Debrecen. First, disease activity data of poly (PM) - and dermatomyositis (DM) patients were compared. Secondly, by patients with active disease, the correlations between the components of Disease Activity Core Set Measures were studied.

Methods: During the three-year follow-up period, at every single visit, authors evaluated disease activity using factors of Disease Activity Core Set Measures and patients filled Health Assessment Questionnaire (HAQ). Only those patients who appeared at least three times during the follow-up period were selected. Statistical analysis was made using SPSS 17.0 statistical software.

Results: Collection of data from 219 patients (age: 44.93 years, female: male ratio 2.98:1) happened in a total of 1101 outpatient visits. The 107 PM patients had statistically significant higher physician and patient visual analogue (VAS) and myositis disease activity assessment tools scores than the $61 \mathrm{DM}$ patients, with the exception of cardiovascular disease activity. Activity of skin lesions was significant higher in DM patients. By the 44 patients, who had an active disease, the MMT scores gave significant negative correlations with HAQ scores $(R=-0.536$ and $p<0.001)$ and $C K$ levels $(R=-0.387$ and $p<0.001)$. The physician and patient VAS gave strong negative correlations with MMT scores $(R=-0.714$ and $p<0.001$ and $R=-0.730$ and $p<0.001$, respectively) and positive correlations with $H A Q$ scores and CK levels (physician VAS vs. $H A Q R=0.691$ and $p<0.001$ patient VAS vs. HAQ $R=0.629$ and $p<0.001$; physician VAS vs. $C K R=0.622$ and $p<0.001$; patient VAS vs. CK $R=0.615$ and $p<0.001$ )

Conclusions: As far as we know, this is the first study which compares the activities of PM and DM patients based on the IMACS system. According to our data, we can conclude that PM patients had more severe muscle symptoms and extramuscular manifestations. Secondly, as seen in other previous studies, calculations in patients with active myositis showed a clear correlation between the most important components of Disease Activity Core Set Measures.

Disclosure of Interest: None declared

DOI: 10.1136/annrheumdis-2018-eular.2220

\section{SAT0498 ABNORMAL ELECTROCARDIOGRAPHIC FINDINGS IN A SCANDINAVIAN COHORT OF PATIENTS WITH IDIOPATHIC INFLAMMATORY MYOPATHIES}

S.S. Korsholm ${ }^{1}$, M. Dastmalchi ${ }^{2}$, A.C. Diederichsen ${ }^{3}$, D.C. Andersson ${ }^{4}$, I. E. Lundberg ${ }^{2}$, N. Witting ${ }^{5}$, M.E. Krogager ${ }^{5}$, T. Friis ${ }^{6}$, S. Jacobsen ${ }^{7}$, J.B. Knudsen ${ }^{1}$, L.P. Diederichsen ${ }^{1} .{ }^{1}$ Department of Rheumatology, Odense University Hospital, Odense C, Denmark; ${ }^{2}$ Department of Rheumatology, Karolinska University Hospital, Stockholm, Sweden; ${ }^{3}$ Department of Cardiology, Odense University Hospital, Odense C, Denmark, ${ }^{4}$ Dept. of Physiology and Pharmacology, Karolinska Institute, Stockholm, Sweden; ${ }^{5}$ Department of Neurology, Copenhagen University Hospital; ${ }^{6}$ Dept. of Autoimmunology and Biomarkers, Statens Serum Institut, ${ }^{7}$ Department of Rheumatology, Copenhagen University Hospital, Copenhagen, Denmark

Background: Idiopathic inflammatory myopathies (IIMs) are characterised by progressive muscle weakness and muscle fatigue. IIMs frequently appear with other organ involvement, e.g. the heart. Cardiac affection is often subclinical but associated with poor prognosis which makes early detection critical.

Presence of autoantibodies is common in IIMs and autoantibodies are important biomarkers to confirm the diagnosis of IIMs. Autoantibodies may even predict organ involvement, e.g. the common myositis specific autoantibody anti-Jo1, which is a marker of lung involvement. So far, an autoantibody predicting cardiac involvement has yet to be identified.

Objectives: The aim of this study was to identify and estimate presence of cardiac involvement detected by electrocardiography (ECG) and to evaluate possible associations between ECG changes and autoantibodies in a Scandinavian cohort of patients with IIMs.

Methods: In a Scandinavian cross-sectional study, 241 patients with polymyositis (PM), dermatomyositis (DM), or inclusion body myositis (IBM) and 48 healthy controls (HCs) were investigated by ECG, basic cardiovascular assessments, and 